\title{
Patient State Index versus Bispectral Index in cirrhotic patients and non-cirrhotics undergoing hepatic resection: A controlled randomized study
}

\author{
Khaled Ahmed Yassen, ${ }^{1}$ Eman Mohammed Badawy, ${ }^{1}$ Maha Lotfy EL Sheikh, \\ Safa Mohamed Helal, ${ }^{2}$ Ahmed AbdelRaouf Metwally. ${ }^{2}$ \\ Anaesthesia Departments of Liver Institute ${ }^{1}$ and Faculty of Medicine, ${ }^{2}$ \\ Menoufia University, Shebeen Elkom City, Egypt
}

\section{Introduction}

Liver resection is a lengthy operation with greater risk of hemodynamic instability during surgery, to avoid intraoperative awareness; Patient State Index (PSI) and Bispectral Index (BIS) monitor anesthesia depth with different algorithms.

\section{Purpose}

> Primary goal is to test PSI\&BIS agreement in 2 different groups:

1. Healthy volunteers undergoing right hepatotomy for living donor liver transplantation.

2. liver cirrhotic patients undergoing major liver resection.

Secondarily their sevoflurane (Sevo) consumption and tolerance to electrocautery interferences.

\section{Methods}

- PSI (Masimo, USA) (Target depth: 25-50) or BIS (Aspect, USA) (Target depth: 40-60). Both forehead sensors were applied simultaneously.

- Anaethesist when monitoring PSI was blinded to BIS and vice versa.

\section{- 4 subgroups: Cirrhotic (PSI)} $(n=15)$, Cirrhotic (BIS) $(n=15)$, Non-cirrhotic (PSI) $(n=15)$ and Non-cirrhotic (BIS) $(n=15)$.

- PSI, BIS values, end-tidal (ET) Sevo concentration (\%) monitored perioperative.

\section{Results}

60 patients, the median age was 56 years old among cirrhotics and 30 years old among non-cirrhotic with similar operation times in all studied group.

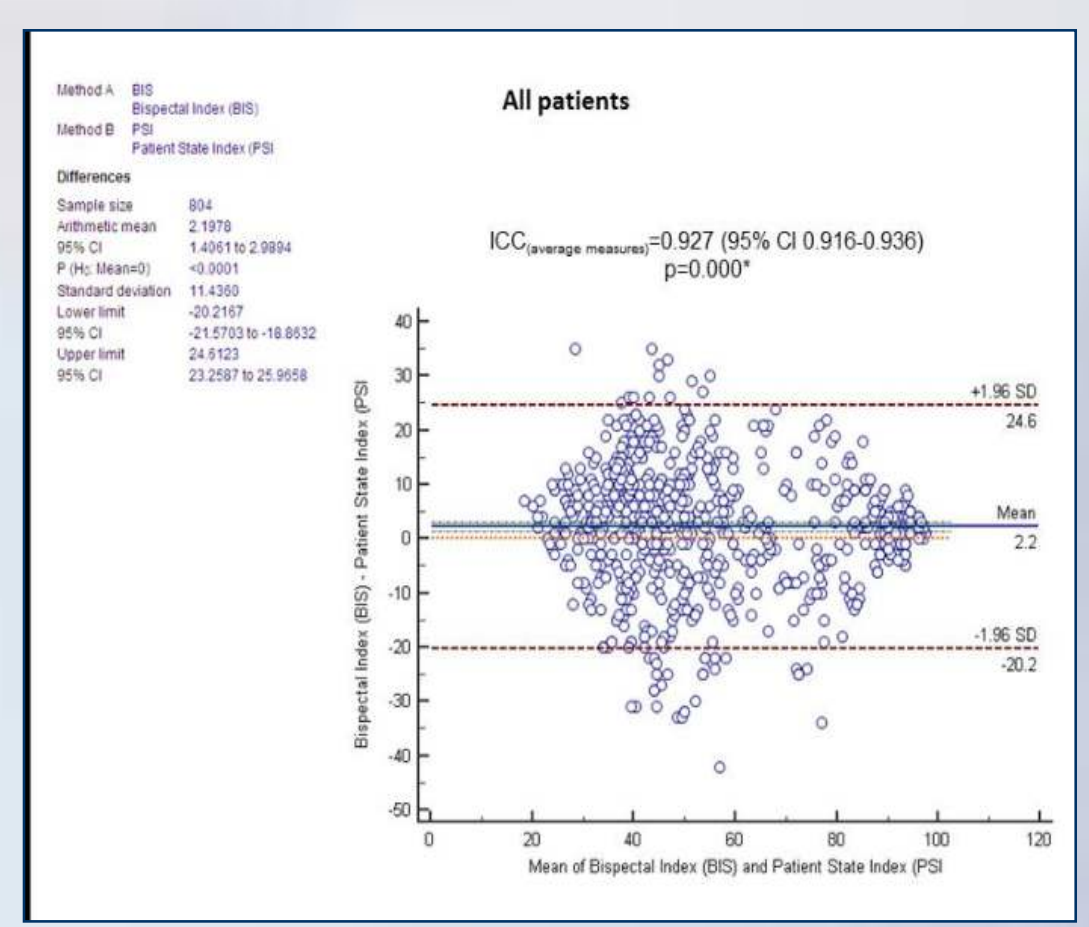

Bland \& Altman analysis showed an overall mean bias difference of 2.19 and $95 \%$ confidence $(1.40-2.98)$, $p<0.0001$. ( $n=804$ pairs $)$

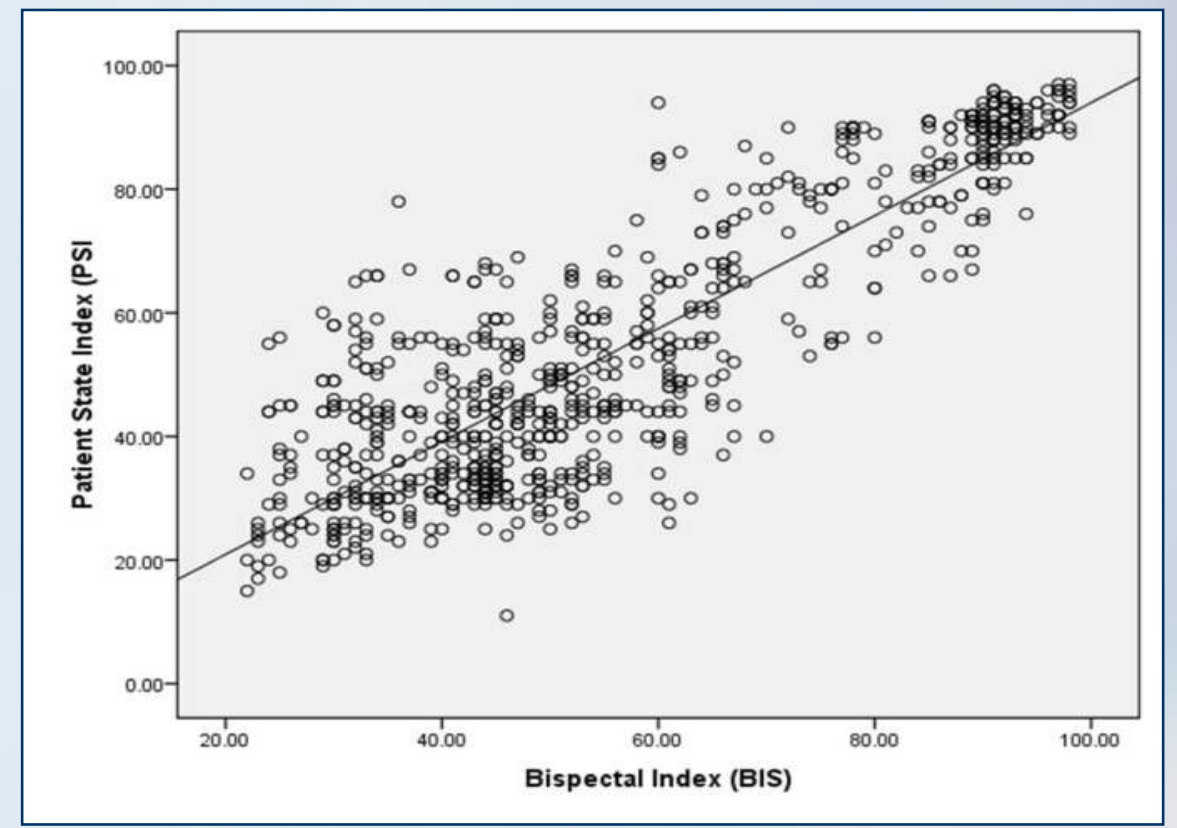

An overall moderate degree of correlation between PSI and BIS (Kendall's tau b= $0.604, p<0.001$. ( $n=804$ pairs)
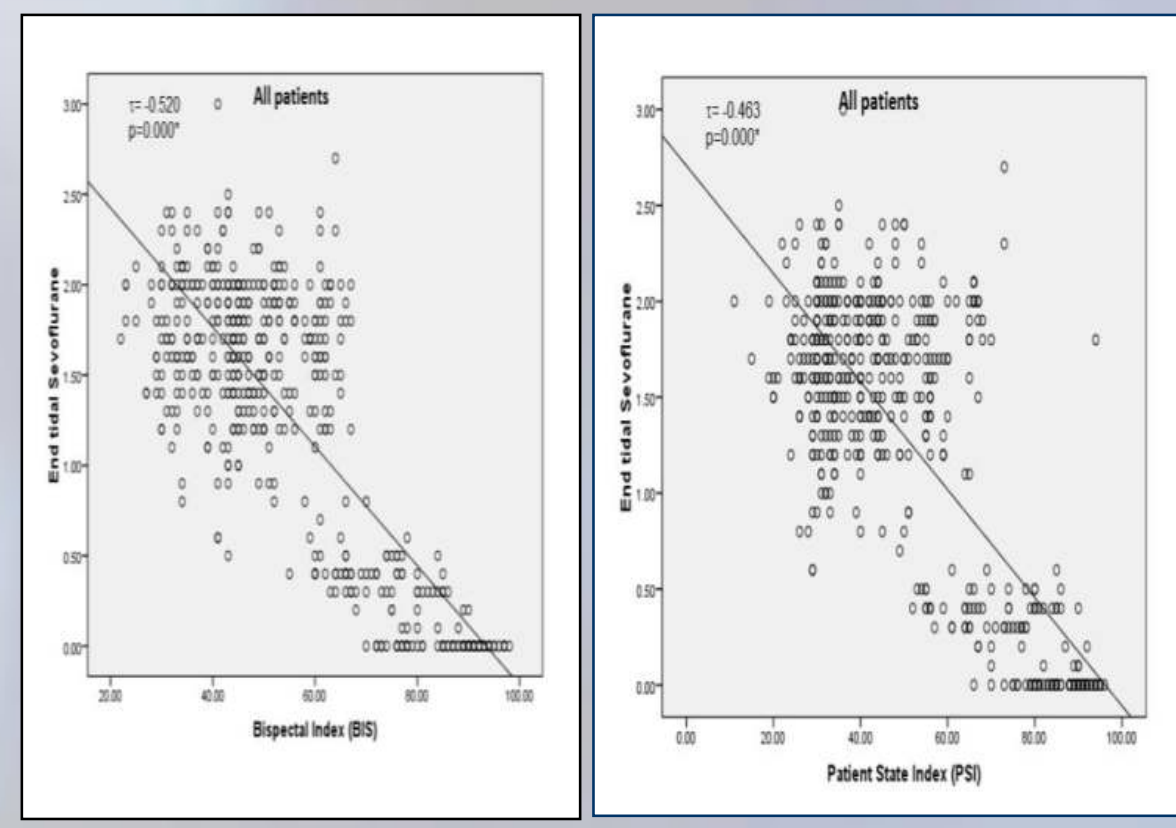

Both PSI \& BIS negatively correlated with ET Sevo in all patients (Kendall's tau $b=-0.463, p=0.00)$ \& (Kendall's tau $\mathrm{b}=-0.520, \mathrm{p}=0.000$ ) respectively.

\begin{tabular}{|l|c|c|c|}
\hline & PSI & BIS & $\begin{array}{c}\text { Test of } \\
\text { significance } \\
\text { (p value) }\end{array}$ \\
\hline $\begin{array}{l}\text { Diathermy } \\
\text { interference } \\
\text { No } \\
\text { Yes }\end{array}$ & $96.67 \%$ & $31.67 \%$ & $\begin{array}{l}Z=7.4246 \\
p=0.00 *\end{array}$ \\
\hline
\end{tabular}

PSI was least affected by electrocautery vs. BIS

PSI guided Sevo consumption (ml) was not different from BIS in cirrhotics (65.67 \pm 31.60 vs. $68.47 \pm 27.63, p=0.983)$ and non-cirrhotics $(41.13 \pm 37.45$ vs.47.27 $\pm 34.80, p=0.338)$, respectively.

\section{Conclusion}

The agreement between PSI and BIS during surgery is excellent with healthy or cirrhotic livers patients. Both were able to monitor trends of anaesthesia 